\title{
Acceleration of ultra-high-energy cosmic rays by local supermassive black hole candidates
}

\author{
Arman Tursunov ${ }^{a, b, *}$ on behalf of the CREDO Collaboration \\ (a complete list of authors can be found at the end of the proceedings) \\ ${ }^{a}$ Research Centre for Theoretical Physics and Astrophysics, Institute of Physics, \\ Silesian University in Opava, Bezručovo nám. 13, CZ-74601 Opava, Czech Republic \\ ${ }^{b}$ Bogoliubov Laboratory for Theoretical Physics, Joint Institute for Nuclear Research, \\ Joliot-Curie street 6, 141980 Dubna, Russia \\ E-mail: arman.tursunov@physics.slu.cz
}

The origin and acceleration mechanism of ultra-high-energy cosmic rays (UHECR) with energy exceeding the GZK-cutoff limit remain unknown. It is often speculated that supermassive black holes (SMBHs) located at the centers of many galaxies can serve as possible sources of UHECR. This is also supported by recent observations of high-energy neutrinos from blazar, as neutrinos are the tracers of UHECR. In this contribution, we explore the capabilities of nearby SMBHs (located within $100 \mathrm{Mpc}$ distance) to accelerate UHECR of certain energy and composition by the novel, ultra-efficient regime of the magnetic Penrose process, in which protons and ions are energized near SMBH by the ionization or decay of low-energy neutral particles, such as e.g. a hydrogen ionization or neutron beta-decay. Extreme conditions around SMBHs increase chances for engagement of the accelerated UHECR in the production of the cosmic ray ensembles (CRE), i.e. a group of correlated two or more cosmic ray particles, including photons with the same parent particle or a common primary interaction vertex. We discuss the unique signatures of UHECR and CRE produced around SMBHs and potentially observable with a global network of detectors, as proposed by the Cosmic-Ray Extremely Distributed Observatory - CREDO.

$37^{\text {th }}$ International Cosmic Ray Conference (ICRC 2021)

July 12 th - 23rd, 2021

Online - Berlin, Germany

\footnotetext{
${ }^{*}$ Presenter
} 


\section{Introduction}

Supermassive black holes (SMBH) located at the centres of most of galaxies are among the largest energy reservoirs in the Universe. The second law of black hole thermodynamics [1] states that up to $29 \%$ of black hole's total mass-energy is the rotational energy, which is available for extraction, i.e. can be transformed into energy of accelerated particles. For SMBH with typical mass of $M=10^{9} M_{\odot}$, the extractable energy is of the order of $10^{74} \mathrm{eV}$. It is therefore most pertinent to tap these enormous sources most effectively and ultra efficiently, as well as to search for the observational signatures of the energy extraction mechanisms. Multiwavelength and multimessenger observations related to both stellar mass and supermassive black hole candidates show no convincing indication of any deviation of the spacetime around these objects from that of the rotating Kerr metric, which states that any astrophysical black hole can be well described by only two parameters, its mass $M$ and spin $a$. Therefore, hereafter by SMBH candidates we bear in mind the rotating black hole with two measurable parameters $M$ and $a$.

Ultra-high-energy cosmic rays (UHECR) are the most energetic among the particles detected on Earth, whose energies may exceed $10^{20} \mathrm{eV}$. Anisotropy studies in arrival directions of primary cosmic rays clearly indicate the extragalactic origin of UHECRs at energies $>10^{18} \mathrm{eV}$ [2]. Allparticle energy spectrum exhibits the existence of two knees at $10^{15.5} \mathrm{eV}$ and $10^{17.5} \mathrm{eV}$ with significant lowering of flux and ankle starting at around $10^{18.5} \mathrm{eV}$ with flattening in the spectrum. At energies exceeding $\sim 10^{19.5} \mathrm{eV}$ for protons, the maximum energy of primary $\mathrm{CR}$ is bounded if the propagation distance from the origin is greater than $\sim 100 \mathrm{Mpc}$ [3], arising due to interaction of charged particles with the SMB photons. Detection of UHECRs at energies greater than GZK-cutoff limit in both northern and southern hemispheres may imply the necessity of search of powerful extragalactic accelerators within the distance $<100 \mathrm{Mpc}$ from the Solar system. There have been many attempts to explain the origin of highest-energy cosmic rays. Among astrophysical scenarios one can mention shock acceleration in relativistic jets [4]. Recently, another acceleration scenario has been suggested [5], which attempted to explain UHECRs by the ionization of the neutral matter in the vicinity of magnetized supermassive black holes (SMBH). In this scenario, the energy of cosmic rays comes in expense of rotational energy of SMBH. In this contribution, we select two dozens of nearby SMBH candidates in order to investigate their UHECR acceleration capabilities.

\section{Energy extraction from rotating black holes}

Roger Penrose in 1969 pointed out [6] the possibility of a test particle moving inside the ergosphere of rotating Kerr black hole to have negative energy with respect to a static observer at infinity, while locally measured energy would remain positive. Using this fact Penrose proposed the first mechanism of the energy extraction from rotating black holes by the fragmentation of a freely falling particle inside the ergosphere into two particles. If one of the fragments attains negative energy, eventually falling into black hole, another one may come out from the ergosphere with energy exceeding the energy of mother particle. Infall of negative energy into black hole is equivalent to the extraction of black hole's rotational energy. Efficiency of Penrose process (defined as the ratio of gained and input energies) was, however, limited to maximum of $21 \%$. Since the black holes are surrounded by a plasma matter that generates their own electromagnetic fields, 
in mid 1980's, the magnetic Penrose process (MPP) has been formulated [7, 8] with interacting particles being charged and black hole being magnetized. It was shown that the efficiency of this process can exceed 100\% due to interaction of charged particles with magnetic field surrounding black hole. Recently, it was shown $[5,9,10]$ that MPP works in three regimes of efficiencies, namely, low, moderate and ultra. In the latter case, the efficiency can be as large as $10^{13} \%$ when applied to typical SMBHs, allowing the direct application of this mechanism for the explanation of UHECRs.

Twisting of magnetic field lines due to black hole's rotation generates electric field component that leads to the non-zero potential difference between the event horizon and infinity. This causes a selective accretion into black hole until the electric potential in a local frame is neutralized, i.e. when $A^{t}=0$ (time component of the four-vector potential of the electromagnetic field). It's covariant component $A_{t}$ is, however, remains non-zero due to the frame-dragging effect. In the black hole vicinity, where the gravity plays a leading role, one can use natural assumption of stationarity and axial symmetry of external magnetic field that is sharing the background symmetries of the Kerr metric spacetime. This assumption is relevant for magnetic field of any origin, wich has a strength sattisfying the condition $B<<10^{18} \mathrm{G}$ for stellar mass black holes and $B<<10^{11}$ for SMBHs [11]. In realistic cases, typical strength of magnetic field for stellar mass black holes vary from few Gauss up to $10^{8} \mathrm{G}$, while for SMBH it is of the order of $10^{4} \mathrm{G}[12,13]$. Therefore, independently from the shapes of magnetic field lines, any astrophysical magnetic field can be considered as a weak test field. However, as we show below, the effect of magnetic field on the motion of charged particles is crucially important.

\subsection{Formalism of the acceleration process}

Let us now consider the motion of a test charged particle with the mass $m$ and the charge $q$. Symmetries of Kerr metric imply allows one to find two conserved quantities (energy and angular momentum), which are the components of the canonical four-momentum $P_{\mu}=m u_{\mu}+q A_{\mu}$ :

$$
E=-P_{t}=m u_{t}+q A_{t}, \quad L=P_{\phi}=m u_{\phi}+q A_{\phi},
$$

where $u^{\mu}$ is the particle's four-velocity. Dynamics of charged particles around Kerr black hole in presence of magnetic field has been widely studied in past; we mention some of previous studies: $[11,14-16]$. Now, we consider the split of a particle $A$, into two charged fragments $B$ and $C$ in the black hole ergosphere at the equatorial plane. The conservation laws before and after split can be written as follows

$$
E_{A}=E_{B}+E_{C}, \quad L_{A}=L_{B}+L_{C}, \quad q_{A}=q_{B}+q_{C}, \quad m_{A} \geq m_{B}+m_{C} .
$$

If one of the particles after split, e.g. particle $B$, attains negative energy, the particle $C$ comes out with energy exceeding the energy of incident particle $A$ in expense of rotational energy of the black hole.

Skipping routine calculations that can be found in [9], we focus on the conditions, for which the energy extraction efficiency grows ultra high. We fix the plane of the motion of the incident particle $A$ to the equatorial plane $\theta=\pi / 2$ and assume that the particle $A$ is neutral, splitting into 
two charged fragments. Then, after several algebraic manipulations and keeping only the leading terms, one can arrive to the following simple expression for the energy of escaping ionized particle

$$
E_{C} \approx \frac{q_{C}}{m_{A}} A_{t}^{i o n} E_{A}
$$

where $A_{t}^{i o n}$ is the time-component of the covariant form of the electromagnetic four-potential calculated at the ionization (decay) point of the incident neutral particle $A$. Note that $A_{t}^{i o n}$ is nonzero in the black hole vicinity due to the frame-dragging effect [17]. The energy in this mechanism can grow ultra-high under realistic conditions [5].

\subsection{Ionization \& maximal energy of accelerated particles}

In general, magnetic field has complicated structure in vicinity of the event horizon, however in a small fraction of a space where split occurs one can consider the field to be approximately uniform [17]. In this case, for the demonstration purposes let us consider the ionization of an arbitrary neutral atom with energy $E=m_{A} c^{2} \sim A \times 10^{9} \mathrm{eV}$ in the vicinity of a typical SMBH. In this case, after the ionization we obtain the following estimate of the energy of an escaping ion

$$
E_{\text {ion }} \approx 10^{20} \mathrm{eV} \frac{Z}{A} \frac{B}{10^{4} \mathrm{G}} \frac{M}{10^{9} M_{\odot}},
$$

where $Z$ and $A$ are the atomic and mass numbers, respectively. For example, in the process of the neutron beta decay, $n^{0} \rightarrow p^{+}+e^{-}+\bar{v}_{\mathrm{e}}$, the energy of the accelerated proton is of the order of $E_{\mathrm{p}+} \approx 10^{20} \mathrm{eV}$. Thus, the model predicts a possible acceleration of protons of the energy of $E_{\mathrm{p}}$ exceeding $10^{20} \mathrm{eV}$ for typical SMBH of mass $M \sim 10^{9} M_{\odot}$ and magnetic field $B \sim 10^{4} G$. Here, we take decay point at $r_{\text {ion }}=r_{g}$, i.e. far enough from the event horizon of rotating black hole, so that the ionized particles are able to escape from the inner region of SMBH. It is important to note, that escaping particle after ionization or decay is more likely a positively charged particle (proton or ion) in the astrophysically favourable cases. This is due to the reason that the induced black hole charge is more likely positive in realistic scenarios $[17,18]$. Numerical modelling of the ionization process in the black hole vicinity can be found in [5].

\section{Mean energy of UHECR protons accelerated by the nearby SMBH candidates}

Standard model predicts an upper limit on the propagation distance of UHECR for a given energy due to the so-called GZK-cutoff $[19,20]$. The maximum distance for UHECR protons that are able to reach the Earth is estimated to be $<100 \mathrm{Mpc}$ [3] with the corresponding energy of protons reaching our Galaxy limited to $5 \times 10^{19} \mathrm{eV}$. Detection of UHECR events with energies greater than GZK-cutoff limit leaves open questions on the origin, propagation and composition of cosmic rays at the highest energies. If UHECRs are constituted by heavier nucleons, interaction of high-energy ions with intergalactic radiation leads to excitation of nuclei and their photodisintegration and fragmentation up to protons and neutrons due to internal resonances if the energy of nucleus is about $10^{20} \mathrm{eV}$. This implies the importance of GZK and photodisintegration phenomena in the propagation of UHECR. Being electrically charged, UHECR also inevitably interact with magnetic fields both in the acceleration zone and along the propagation distance. Although suppression of 
Table 1: A list of selected $25 \mathrm{SMBH}$ candidates with measured masses $M$ and distances $d$, estimated spins $a$ and magnetic field strengths $B$ and predicted mean energies of proton $E_{p+}^{\text {mean }}$ escaping from corresponding source after the ionization in the vicinity of SMBH.

\begin{tabular}{c|c|c|c|c|c}
\hline SMBH & $\log \left(M / M_{\odot}\right)$ & $\operatorname{Spin} a$ & $\mathrm{~d}(\mathrm{Mpc})$ & $\log (B / 1 \mathrm{G})$ & $\log \left(E_{p+}^{\text {mean }} / 1 \mathrm{eV}\right)$ \\
\hline \hline Sgr A & 6.63 & 0.5 & 0.008 & 2 & 15.64 \\
\hline NGC 1052 & 8.19 & $\lesssim 1$ & 19 & 4.8 & 20.11 \\
\hline NGC 1068 / M77 & 6.9 & $\lesssim 1$ & 15 & 4.54 & 18.56 \\
\hline NGC 1365 & 6.3 & $\lesssim 1$ & 17.2 & 4.70 & 18.12 \\
\hline NGC 2273 & 6.9 & 0.97 & 29 & 4.58 & 18.41 \\
\hline NGC 2787 & 7.6 & $\lesssim 1$ & 8 & 3.73 & 18.45 \\
\hline NGC 3079 & 6.4 & $\lesssim 1$ & 22 & 4.06 & 17.58 \\
\hline NGC 3516 & 7.4 & 0.64 & 42 & 4.88 & 19.37 \\
\hline NGC 3783 & 7.5 & 0.98 & 41 & 4.15 & 18.77 \\
\hline NGC 3998 & 8.9 & 0.54 & 15 & 3.58 & 19.52 \\
\hline NGC 4151 & 7.8 & 0.84 & 14 & 4.6 & 19.53 \\
\hline NGC 4258 / M106 & 7.6 & 0.38 & 8 & 4.14 & 18.65 \\
\hline NGC 4261 & 8.7 & $\lesssim 1$ & 32 & 3.51 & 19.33 \\
\hline NGC 4374 / M84 & 9 & 0.98 & 20 & 3 & 19.12 \\
\hline NGC 4388 & 6.9 & 0.51 & 18 & 5.19 & 19.11 \\
\hline NGC 4486 / M87 & 9.7 & $\lesssim 1$ & 17 & 2.84 & 19.66 \\
\hline NGC 4579 & 8 & 0.82 & 18 & 4.11 & 19.23 \\
\hline NGC 4594 & 8.8 & 0.6 & 11 & 3.18 & 19.05 \\
\hline NGC 5033 & 7.2 & 0.68 & 20 & 4.47 & 18.77 \\
\hline NGC 5194 / M51 & 6.0 & 0.57 & 8 & 4.51 & 17.57 \\
\hline MCG-6-30-15 & 7.3 & 0.98 & 33 & 4.74 & 19.16 \\
\hline NGC 5548 & 7.8 & 0.58 & 75 & 4.48 & 19.34 \\
\hline NGC 6251 & 8.8 & $\lesssim 1$ & 102 & 3.70 & 19.62 \\
\hline NGC 6500 & 8.6 & $\lesssim 1$ & 43 & 3.60 & 19.32 \\
\hline IC 1459 & 9.4 & $\lesssim 1$ & 31 & 3.20 & 19.72 \\
\hline
\end{tabular}

energy due to synchrotron radiation in a Galactic and intergalactic magnetic fields is relatively small, UHECRs can loose sufficient amount of their energies in the source regions where magnetic fields can be considerably large [15].

Taking into account GZK, photodisintegration and synchrotron energy loss mechanisms, we calculate the mean energies of the cosmic ray protons accelerated by the nearby sources. In the Table 1, we present a list of 25 nearby SMBH candidates located within the distance of $\sim 100 \mathrm{Mpc}$ and predict the mean values of UHE protons for each source produced in the above described process. We emphasize that it is not an exhaustive list, but intended mainly to bring an idea on capability of SMBH candidates with the state of the art measurements of masses, spins and magnetic fields to produce the highest energy particles. The masses $M$ and distances $d$ in Table 1 are based on 
the observations and direct measurements, while spin $a$ and magnetic field $B$ estimates are obtained by well-established methods based on the measurements of luminosity, spectra and polarimetric parameters of the sources. The data used in the table are taken from [12, 13, 21, 22] and references therein. Last column on the right demonstrates the predictions of the model on the mean energies of protons $E_{p+}^{\text {mean }}$ accelerated by each given SMBH candidate source.

\section{Discussion \& conclusions}

Supermassive black holes (SMBH) are among the most energetic objects in the Universe, while ultra-high-energy cosmic rays (UHECR) are the most energetic among particles detected on Earth. In the same time, in the age of multi-wavelength and multi-messenger astronomy, black holes and their environments were not yet probed with the cosmic ray messengers. Extreme conditions in the environments of SMBH increase chances for engagement of the accelerated UHECR in the production of the cosmic ray ensembles (CRE), i.e. a group of correlated two or more cosmic ray particles, including photons with the same parent particle or a common primary interaction vertex. Experimental searches for cosmic-ray correlations have been realized on different scales with the highest energy events observed by the leading collaborations: Pierre Auger Observatory and Telescope Array, as well as the projects with distributed arrays of detectors, such as e.g. CHICOS, ALTA, CZELTA, GELATICA, EEE, and LAAS. Time correlation of registered showers was studied at the distances from $100 \mathrm{~m}$ to $7000 \mathrm{~km}$, and in some cases evidence for unexpected coincidences have been found, however without any convincing follow-up studies and data taking campaigns, which is hard without a global coordination. Recently the idea of looking for large scale correlations in a general and global way took shape of the Cosmic Ray Extremely Distributed Observatory (CREDO) Collaboration (see, recent review [23]) formalized in September 2019. CREDO is meant to be a multi-technique (different detector types) and doubly open (for both data upload and offering access) infrastructure enabling a global research programs concerning radiation (both cosmic and terrestrial), with a number of multi-messenger, multi-mission and transdisciplinary opportunities.

In this contribution we studied the energy transfer from nearby rotating SMBHs to UHECRs in the process of ionization of neutral particles skirting close to black holes. Rotation of a black hole in external magnetic field gives a rise to an induced electric field due to frame-dragging effect and subsequent net charging of the black hole. The best efficiency of energy extraction can be achieved in the processes involving ionization of neutral matter at the event horizon scales, in which the ionized particle in addition to its mass energy obtains also the electromagnetic contribution. Due to certain alignment of the magnetic field lines produced by the co-rotating plasma it is more plausible that the induced black hole charge is positive, which accelerates protons and ions to ultra-high energies along the symmetry axis. Other fragments, the electrons eventually collapse into the black hole decreasing thus, the black hole's angular momentum.

We have selected 25 nearby SMBH candidates located within $100 \mathrm{Mpc}$ distance with estimated masses, spins and magnetic fields in their vicinities. Calculating the mean proton energy resulting from the ionization, we presented results in a table 1, where we have shown several plausible candidates for UHECR sources at energies greater than GZK-limit. We also applied our model to Sgr A* located at the center of our Galaxy, which is the best known SMBH candidate. Remarkably, 
we found that $\mathrm{Sgr} \mathrm{A}^{*}$ can serve as a PeVatron with proton energy at the level of $5 \times 10^{15} \mathrm{eV}$. This implies that the Galactic centre can potentially contribute to the sharpness of the knee of the cosmic ray spectrum. One can also note that the model predicts similar orders of magnitudes of energies of accelerated protons for selected SMBH candidates, which have different masses, locations and magnetic field estimates. The mean proton energy averaged over all selected extragalactic sources appears to be around $10^{19} \mathrm{eV}$. Existence of many sources at such energies can be potentially relevant in the interpretation of the ankle of the cosmic ray spectrum, though, the expected spectrum from particular nearby objects are yet to be determined.

\section{Acknowledgments}

A.T. thanks the Institute of Physics of Silesian University in Opava for institutional support. We acknowledge the support from the International Visegrad Fund's Visegrad Grant No. 21920298.

\section{References}

[1] J. M. Bardeen, B. Carter, and S. W. Hawking. The four laws of black hole mechanics. Communications in Mathematical Physics, 31:161-170, June 1973.

[2] Pierre Auger Collaboration. An Indication of Anisotropy in Arrival Directions of Ultra-highenergy Cosmic Rays through Comparison to the Flux Pattern of Extragalactic Gamma-Ray Sources. Astrophysical Journal Letters, 853:L29, February 2018.

[3] F. W. Stecker. Effect of Photomeson Production by the Universal Radiation Field on HighEnergy Cosmic Rays. Phys. Rev. Lett. , 21(14):1016-1018, September 1968.

[4] R. D. Blandford. Acceleration of Ultra High Energy Cosmic Rays. Physica Scripta Volume $T, 85: 191-194,2000$.

[5] A. Tursunov, Z. Stuchlík, M. Kološ, N. Dadhich, and B. Ahmedov. Supermassive Black Holes as Possible Sources of Ultrahigh-energy Cosmic Rays. The Astrophysical Journal , 895(1):14, May 2020.

[6] R. Penrose. Gravitational Collapse: the Role of General Relativity. Nuovo Cimento Rivista Serie, 1, 1969.

[7] S. M. Wagh, S. V. Dhurandhar, and N. Dadhich. Revival of the Penrose process for astrophysical applications. The Astrophysical Journal , 290:12-14, March 1985.

[8] S. Parthasarathy, S. M. Wagh, S. V. Dhurandhar, and N. Dadhich. High efficiency of the Penrose process of energy extraction from rotating black holes immersed in electromagnetic fields. The Astrophysical Journal , 307:38-46, August 1986.

[9] A. Tursunov and N. Dadhich. Fifty Years of Energy Extraction from Rotating Black Hole: Revisiting Magnetic Penrose Process. Universe, 5(5):125, May 2019. 
[10] N. Dadhich, A. Tursunov, B. Ahmedov, and Z. Stuchlík. The distinguishing signature of magnetic Penrose process. Monthly Notices of the Royal Astronomical Society, 478:L89L94, July 2018.

[11] A. Tursunov, Z. Stuchlík, and M. Kološ. Circular orbits and related quasiharmonic oscillatory motion of charged particles around weakly magnetized rotating black holes. Phys. Rev. D, 93(8):084012, April 2016.

[12] R. A. Daly. Black Hole Spin and Accretion Disk Magnetic Field Strength Estimates for More Than 750 Active Galactic Nuclei and Multiple Galactic Black Holes. The Astrophysical Journal , 886(1):37, November 2019.

[13] M. Yu Piotrovich, A. G. Mikhailov, S. D. Buliga, and T. M. Natsvlishvili. Determination of magnetic field strength on the event horizon of supermassive black holes in active galactic nuclei. Monthly Notices of the Royal Astronomical Society , 495(1):614-620, May 2020.

[14] Z. Stuchlík and M. Kološ. Acceleration of the charged particles due to chaotic scattering in the combined black hole gravitational field and asymptotically uniform magnetic field. European Physical Journal C, 76:32, January 2016.

[15] A. Tursunov, M. Kološ, Z. Stuchlík, and D. V. Gal'tsov. Radiation Reaction of Charged Particles Orbiting a Magnetized Schwarzschild Black Hole. The Astrophysical Journal, 861:2, July 2018.

[16] Z. Stuchlík, M. Kološ, J. Kovář, P. Slaný, and A. Tursunov. Influence of Cosmic Repulsion and Magnetic Fields on Accretion Disks Rotating around Kerr Black Holes. Universe, 6(2):26, January 2020.

[17] R. M. Wald. Black hole in a uniform magnetic field. Phys. Rev. D , 10:1680-1685, September 1974.

[18] M. Zajaček, A. Tursunov, A. Eckart, and S. Britzen. On the charge of the Galactic centre black hole. Monthly Notices of the Royal Astronomical Society , 480:4408-4423, November 2018.

[19] K. Greisen. End to the cosmic ray spectrum? Phys. Rev. Lett., 16:748-750, 1966.

[20] G. T. Zatsepin and V. A. Kuzmin. Upper limit of the spectrum of cosmic rays. JETP Lett., 4:78-80, 1966. [Pisma Zh. Eksp. Teor. Fiz.4,114(1966)].

[21] S. S. Doeleman and et al. Jet-Launching Structure Resolved Near the Supermassive Black Hole in M87. Science, 338:355, October 2012.

[22] M. Kino, F. Takahara, K. Hada, K. Akiyama, H. Nagai, and B. W. Sohn. Magnetization Degree at the Jet Base of M87 Derived from the Event Horizon Telescope Data: Testing the Magnetically Driven Jet Paradigm. The Astrophysical Journal , 803:30, April 2015.

[23] P. Homola et al. for the CREDO Collaboration. Cosmic-Ray Extremely Distributed Observatory. Symmetry, 12(11):1835, November 2020. 


\section{Full Authors List: CREDO Collaboration}

Arman Tursunov ${ }^{1,2}$ David E. Alvarez Castillo ${ }^{3,2}$, Alok C. Gupta ${ }^{4}$, Bohdan Hnatyk ${ }^{5}$, Piotr Homola ${ }^{3}$, Marcin Kasztelan $^{6}$, Martin Kološ ${ }^{1}$, Peter Kovacs ${ }^{7}$, Bartosz Lozowski ${ }^{8}$, Mikhail V. Medvedev ${ }^{9}, 10$, Alona Mozgova ${ }^{5}$, Michał Niedźwiecki ${ }^{11}$, Matías Rosas ${ }^{12}$, Krzysztof Rzecki $^{13}$, Katarzyna Smelcerz ${ }^{11}$, Karel Smolek ${ }^{14}$, Jarosław Stasielak ${ }^{3}$, Zdeněk Stuchlík ${ }^{1}$, Oleksandr Sushchov ${ }^{3}$, Tadeusz Wibig ${ }^{15}$, Jilberto Zamora-Saa ${ }^{16}$.

${ }^{1}$ Research Centre for Theoretical Physics and Astrophysics, Institute of Physics, Silesian University in Opava, Bezručovo nám. 13, CZ-74601 Opava, Czech Republic.

${ }^{2}$ Bogoliubov Laboratory for Theoretical Physics, Joint Institute for Nuclear Research, Joliot-Curie street 6, 141980 Dubna, Russia.

${ }^{3}$ Institute of Nuclear Physics Polish Academy of Sciences, Radzikowskiego 152, 31-342 Kraków, Poland.

${ }^{4}$ Aryabhatta Research Institue of Observational Sciences (ARIES), Manora Peak, Nainital 263001, India.

${ }^{5}$ Astronomical Observatory of Taras Shevchenko National University of Kyiv, 04053 Kyiv, Ukraine.

${ }^{6}$ National Centre for Nuclear Research, Andrzeja Soltana 7, 05-400 Otwock-Świerk, Poland.

${ }^{7}$ Institute for Particle and Nuclear Physics, Wigner Research Centre for Physics, 1121 Budapest, Konkoly-Thege Miklós út 29-33, Hungary.

${ }^{8}$ Faculty of Natural Sciences, University of Silesia in Katowice, Bankowa 9, 40-007 Katowice, Poland.

${ }^{9}$ Department of Physics and Astronomy, University of Kansas, Lawrence, KS 66045, USA.

${ }^{10}$ Laboratory for Nuclear Science, Massachusetts Institute of Technology, Cambridge, MA 02139, USA.

${ }^{11}$ Department of Computer Science, Faculty of Computer Science and Telecommunications, Cracow University of Technology, Warszawska 24, 31-155 Kraków, Poland.

${ }^{12}$ Liceo 6 Francisco Bauzá, Montevideo, Uruguay.

${ }^{13}$ AGH University of Science and Technology, Mickiewicz Ave., 30-059 Kraków, Poland.

${ }^{14}$ Institute of Experimental and Applied Physics, Czech Technical University in Prague.

${ }^{15}$ University of Łódź, Faculty of Physics and Applied Informatics, 90-236 Łódź, Pomorska 149/153, Poland.

${ }^{16}$ Universidad Andres Bello, Departamento de Ciencias Fisicas, Facultad de Ciencias Exactas, Avenida Republica 498, Santiago, Chile. 